\title{
Fauna de arañas del suelo de una comunidad árida-tropical en Baja California Sur, México
}

\section{Ground surface spider fauna in an arid tropical community in Baja California Sur, Mexico}

\author{
María Luisa Jiménez* y José Gabriel Navarrete \\ Laboratorio de Aracnología y Entomología, Centro de Investigaciones Biológicas del Noroeste, S.C. Apartado postal 128, 23090, La Paz, Baja \\ California Sur, México. \\ *Correspondencia: ljimenez04@cibnormx
}

\begin{abstract}
Resumen. Se describe la temporalidad y diversidad de arañas del suelo del matorral sarcocaule en la región del Cabo en Baja California Sur. Durante 1991-1992 se realizaron colectas semanales por medio de trampas de caída. Se capturaron 4322 ejemplares de 53 especies. Las familias con mayor abundancia fueron Miturgidae (42.2\%), Oonopidae (23.5\%), Caponiidae (8.0\%) y Gnaphosidae (7.9\%). Las especies más abundantes fueron Syspira tigrina Chamberlin (37.1\%); Oonops nov. sp. (13.7\%), Scaphiella hespera Chamberlin (7.8\%) y Tarsonops sternalis Chamberlin (5.2\%), que constituyeron el $63.7 \%$ del total de individuos. Gnaphosidae fue la más rica en especies (11), seguida por Salticidae (7). La riqueza de especies fue constante durante todo el año, con un ligero ascenso en el verano (29) y una ligera disminución en el invierno (24). La diversidad por estación del año se mantuvo en un intervalo de $\mathrm{H}^{\prime}=3.3$-3.7. La abundancia relativa se incrementó en primavera y otoño. La mayor riqueza específica se encontró en otoño. La abundancia relativa y la diversidad de arañas se pueden considerar altas, a pesar del fuerte dominio de la familia Miturgidae. En la mayoría de las especies, la distribución mostró una marcada estacionalidad.
\end{abstract}

Palabras clave: arañas edáficas, Baja California Sur, matorral desértico.

\begin{abstract}
Seasonal distribution, specific richness, and diversity of xeric shrub ground spiders were studied at a site in the Cape Region. Weekly collections of spiders were made in 1991-1992 using pit-fall traps. We captured 4322 specimens in 53 families. Families with the highest number of individuals were: Miturgidae (42\%) Oonopidae (23.5\%), Caponiidae (8.0\%), and Gnaphosidae (7.9\%). The most abundant species were Syspira tigrina Chamberlin (37.1\%); Oonops nov. sp. (13.7\%), Scaphiella hespera Chamberlin (7.8\%), and Tarsonops sternalis Chamberlin (5.2\%), representing $63.7 \%$ of the total specimens captured. Gnaphosidae was the richest in species (11) followed by Salticidae (7). Species richness was nearly constant during all the year, with a small increase in summer (29) and a small decline in winter (24); seasonal diversity was also constant $\left(H^{\prime}=3.3-3.7\right)$. The highest relative abundance occurred in spring and autumn and the highest specific richness was in autumn. The diversity and relative abundance of spiders were high, in spite of the high abundance of Miturgidae. Distribution of the major species had a marked seasonality.
\end{abstract}

Key words: ground spiders, Baja California Sur, desert shrub.

\section{Introducción}

Desde hace varios años, el estudio de las arañas de los desiertos ha adquirido relevancia debido al papel fundamental que dichos organismos desempeñan en estos ecosistemas; junto con otros arácnidos, son el grupo más importante de depredadores en estas zonas (Polis y Yamashita, 1991). En las zonas desérticas, las arañas cazadoras conforman la mayoría del grupo (CloudsleyThompson, 1983); son tan abundantes que llegan a constituir hasta un $87 \%$ del total de las arañas en algunos

Recibido: 27 marzo 2009; aceptado: 14 septiembre 2009 desiertos del mundo (Polis y Yamashita, 1991). En el desierto de Mojave constituyen el $75 \%$ del total de la población de arañas (Allred et al., 1963); en el desierto de Chihuahua, el $80 \%$ (Chew, 1961) y el 59\% en el Valle Coachella (Polis y McCormick, 1986).

La península de Baja California -la segunda más grande del mundo-, por su aislamiento geográfico e historia geológica, aloja una flora y fauna muy interesante. Baja California está considerada un desierto costero frío (Cloudsley-Thompson, 1979), con vegetación muy variada del tipo desierto sonorense (Axelrod, 1979). Después de Veracruz, es el segundo estado de México con el mayor número de especies, agrupando un $14.8 \%$ del 
total registrado para este país (Jiménez, 1996).

El conocimiento de las arañas de esta región es bueno; sin embargo, está limitado a la descripción de especies, mientras que los estudios sobre la ecología del grupo son escasos. Se cuenta solamente con estudios aislados sobre especies asociadas a agroecosistemas (Jiménez y Tejas, 1996); de algunas que se han establecido como residentes en viviendas urbanas (Jiménez, 1998); de otras asociadas a humedales ( Llinas y Jiménez, 2004), y de otras más que forman parte importante en la dieta de insectos, como las avispas Seliphron y Trypoxylon (Jiménez et al., 1992; Jiménez y Tejas 1994; Domínguez y Jiménez, 2008).

En este estudio se describe por primera vez la composición taxonómica de la comunidad de especies de arañas del suelo de una localidad árido-tropical correspondiente al matorral desértico tipo sarcocaule en la región del Cabo, Baja California Sur, y se analiza su distribución a lo largo de un ciclo anual, relacionándola con algunas variables ambientales.

\section{Materiales y métodos}

Área de estudio. El área de estudio se localiza en los $24^{\circ} 5^{\prime} \mathrm{N}$ y $110^{\circ} 21^{\prime}$ O cerca de la bahía de La Paz, Baja California Sur, México, en un predio llamado El Comitán con una extensión de 220 ha (León de la Luz et. al., 1996) (Fig. 1). Esta localidad corresponde a una planicie aluvial formada a partir del Pleistoceno por el acarreo y depósito de fragmentos de rocas graníticas que provienen de la actividad erosiva de la sierra de La Laguna (Hammond, 1954). El suelo es de tipo vermisol háplico, con horizontes débilmente diferenciados, muy pobre en materia orgánica y de textura arenosa (León de la Luz et al., 1996). El clima corresponde a un BW (h') hw(e), es decir, cálido muy seco, con precipitación invernal superior al $10 \%$ del total anual, el invierno es fresco, pero no se registran heladas (García, 1981). Las temperaturas medias extremas se presentan en enero y agosto $\left(18^{\circ}\right.$ y $30^{\circ} \mathrm{C}$ respectivamente) y la temperatura media anual es de $24^{\circ}-26^{\circ} \mathrm{C}$ (SPP, 1981b). La precipitación total del mes más seco (abril-mayo) fue de $0.2 \mathrm{~mm} / \mathrm{mes}$ (carta de humedad del suelo (SPP, 1981a).

La vegetación del área, se encuentra dentro de una planicie costera aluvial y corresponde al matorral sarcocaule, mismo que se caracteriza por la presencia de especies de tallo grueso y voluble y crasicaule de tallo suculento. La vegetación está representada por 136 especies vasculares cuyas especies perennes dominantes son: Fouqueria diguetti, Jatropha cuneata, Stenocereus gummosus, Opuntia cholla, Cyrtocarpa edulis, Atamisquea emarginata, Brusera microphylla, Stenocereus thurberii, Prosopis articulata, entre otras

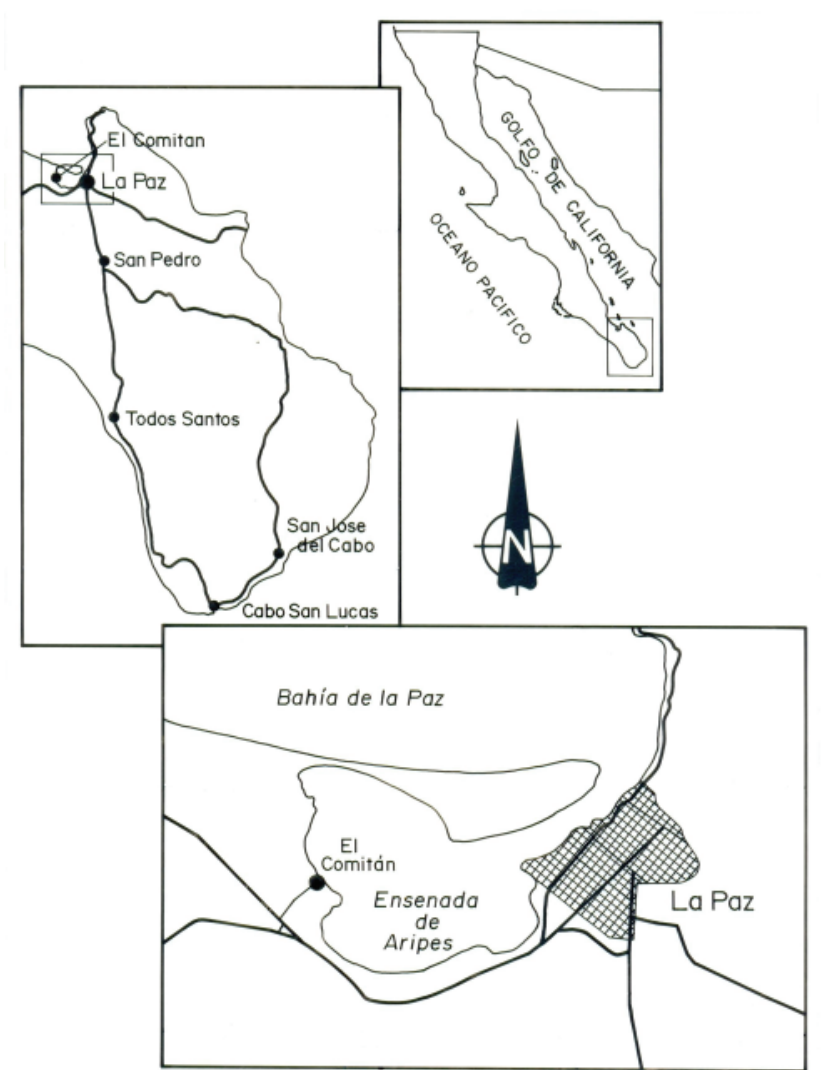

Figura 1. Localización del área de estudio El Comitán, B.C.S, México.

(León de la Luz et al., 1996).

Trabajo de campo. Las colectas se llevaron a cabo de noviembre de 1991 a noviembre de 1992, por medio de trampas fijas de caída (22 en total) que se revisaron semanalmente durante un año, colocadas a lo largo de 2 transectos paralelos, siguiendo el curso de un arroyo de temporal, con un intervalo de $7 \mathrm{~m}$ entre una y otra. Cada trampa consistió en un recipiente recolector de plástico de $13 \mathrm{~cm}$ de ancho por $15.5 \mathrm{~cm}$ de alto, que se enterró al nivel del suelo y en cuyo interior fue colocado un frasco de vidrio $(7 \times 5.5 \mathrm{~cm})$ con una mezcla de alcohol y etilenglicol al $70 \%$ en partes iguales, que actuó como fijador y conservador (modificada de Uetz y Unzicker, 1976). A este recipiente se le colocó un embudo de plástico al que previamente se le cortó la parte más estrecha para encauzar la captura al frasco con el fijador. A todos los recipientes se les cubrió con una red metálica de $22 \times 22 \mathrm{~cm}$, con 4 pequeñas prolongaciones de $6 \mathrm{~cm}$ en las esquinas, para que quedaran sobre el nivel de suelo, lo que permitió el paso libre de los organismos y al mismo tiempo evitó que la hojarasca u otro tipo de material se introdujera en el recipiente. Cada semana se remplazó el frasco con el 
fijador, y el contenido se llevó al laboratorio, donde las arañas fueron separadas y cuantificadas; posteriormente, se identificaron a nivel específico y se colocaron en tubos de vidrio con alcohol etílico al $70 \%$ para su conservación.

Para la determinación de la diversidad de la comunidad, se empleó el índice de Shannon, por utilizarse en estudios de arañas cursoriales (Uetz y Unzicker, 1976; Bultman et al., 1982). La similitud entre las 4 estaciones, fue medida con el índice de Morisita. Se obtuvo la abundancia relativa de cada especie. Los datos de precipitación y temperatura fueron proporcionados por la Estación Meteorológica del CIBNOR.

Todo el material biológico se encuentra depositado en la Colección Aracnológica del Centro de Investigaciones Biológicas del Noroeste (CARCIB), La Paz, Baja California Sur, México.

\section{Resultados}

Se capturaron 4322 ejemplares pertenecientes a 52 especies, 44 géneros y 22 familias de arañas del suelo. En el Cuadro 1 se muestra la composición de especies. La familia con mayor abundancia fue Miturgidae (42.2\%), seguida por Oonopidae $(23.5 \%)$, Caponiidae $(8.0 \%)$, Gnaphosidae (7.9\%) y Salticidae (3.6\%), que totalizan el $85.2 \%$, mientras que las 17 restantes sólo el $15.8 \%$.

Gnaphosidae fue la familia con la mayor riqueza de especies (11), seguida por Salticidae (7), mientras que 9 de las 22 familias registradas en este trabajo (Agelenidae, Homalonychidae, Loxoscelidae, Oecobiidae, Philodromidae, Pholcidae, Plectreuridae, Selenopidae, Dipluridae) estuvieron representadas por 1 especie (Cuadro 1).

Las especies más abundantes fueron Syspira tigrina Chamberlin (37.1\%), Oonops. sp. (13.7\%), Scaphiella hespera Chamberlin (7.8\%) (Oonopidae) y Tarsonops sternalis Chamberlin (5.2\%). Estas 4 especies constituyeron el $63.7 \%$ del total de individuos, mientras que Trachelas speciosus Banks, Kukulcania hibernalis Hentz, Litopyllus realisticus (Chamberlin) y Zelotes monachus Chamberlin destacaron particularmente por su escasez (Cuadro 1).

Del total de individuos capturados, los juveniles fueron los más abundantes (56.8\%), seguida por los machos $(25.5 \%)$ y las hembras $(17.6 \%)$. El radio sexual macho/ hembra fue de 2:1, destacando Promyrmekiaphila sp., por tener el mayor número de machos (Cuadro 1).

La mayor riqueza de especies se observó en los meses de julio (36), abril (33) y septiembre (32) y la menor en enero (22) y octubre (23). Al igual que la similitud, la diversidad por estación del año se mantuvo constante con un ligero aumento en invierno (Cuadro 2).
La frecuencia máxima de individuos capturados ocurrió en primavera y otoño. Al descender la temperatura y aumentar la precipitación, se observó una abundancia mínima durante el invierno, mientras que en el verano, con la temperatura máxima, se registró un ligero decremento de individuos entre los 2 incrementos máximos (Fig. 2). No hubo una correlación estadísticamente significativa con la temperatura y la abundancia de arañas $(\mathrm{r} 2=0.42)$, pero sí con la precipitación $(\mathrm{r} 2=0.79)$.

En la figura 3 se muestra la variación temporal de las familias de arañas más abundantes de esta localidad, destacando Miturgidae, con la mayor abundancia en otoño y un incremento más bajo en invierno, mientras que Oonopidae mostró un aumento en primavera y una disminución en otoño. Caponiidae y Gnaphosidae mantuvieron sus poblaciones casi constantes durante todo el año, pero se observó un ligero aumento de ambas durante el invierno. Salticidae presentó su mayor abundancia en otoño y Thomisidae en invierno. Las Dictynidae fueron más abundantes durante la primavera, y las Oxyopidae durante el otoño.

La presencia de las diferentes especies a través del año se muestran en el Cuadro 3, en el que se pueden observar 2 grupos: 1), especies que se presentaron durante todo el año: T. sternalis, S. tigrina, Dictyna sp, N. pubecens (Banks), Z. monachus, S. hespera Chamberlin, Y. nesophila Chamberlin, O. stylifer Gertsch, O. tridens Brady y Oonops sp., y 2), especies que estuvieron presentes en un sólo mes: Novalena sp., Trachelas speciosus, K. hibernalis, L. realisticus, Zelotes catholicus Chamberlin, Tmarus sp., Metaphidippus sp. 2, Actinoxia sp. Myrmekiaphila sp. y Aphonopelma sp. El resto de las especies se presentaron indistintamente en una o más épocas del año.

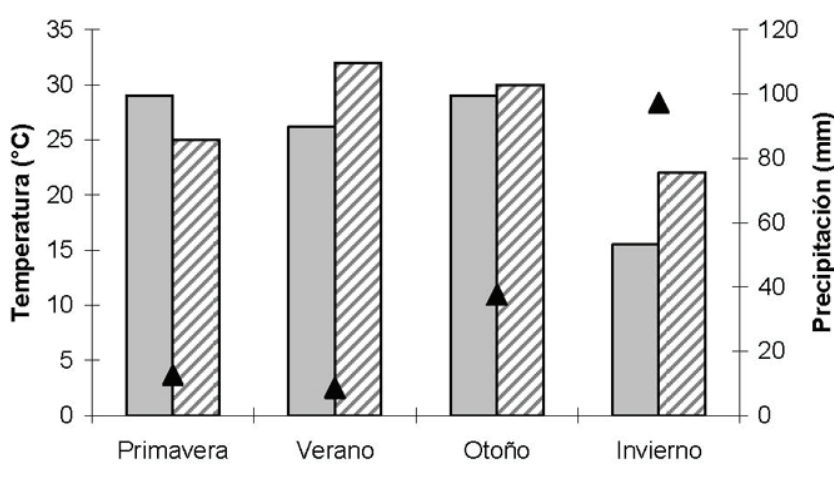

$\square$ Abundancia relativa (\%) $\boldsymbol{\nabla}$ Temperatura $\boldsymbol{\Delta}$ Precipitacion

Figura 2. Abundancia relativa de la aracnofauna (\%) temperatura y precipitación durante el periodo 1991-1992, en El Comitán, B.C.S., México. 
Cuadro 1. Abundancia relativa (\%) y frecuencia de los estadios de arañas del suelo del matorral sarcocaule de El Comitán, B.C.S. México 1991-1992. J = juveniles

\begin{tabular}{|c|c|c|c|c|c|c|}
\hline Familia & Especies & $\%$ & $\hat{0}$ & q & $J$ & Totales \\
\hline Agelenidae & Novalena sp. & 0.06 & 3 & & & 3 \\
\hline \multirow[t]{3}{*}{ Caponiidae } & Tarsonops sectipes Chamberlin & 1.13 & 10 & 5 & 34 & 49 \\
\hline & Tarsonops sternalis (Banks ) & 5.21 & 68 & 137 & 21 & 226 \\
\hline & Orthonops overtus (Chamberlin ) & 1.62 & 32 & 22 & 16 & 70 \\
\hline Clubionidae & Clubiona sp. & 1 & 5 & 3 & 35 & 43 \\
\hline \multirow[t]{2}{*}{ Corinidae } & Trachelas speciosus Banks & 0.02 & 1 & & & 1 \\
\hline & Castianeira dorsata (Banks) & 0.16 & 2 & & 5 & 7 \\
\hline \multirow[t]{2}{*}{ Miturgidae } & Syspira tigrina Chamberlin & 37.1 & 310 & 133 & 1161 & 1604 \\
\hline & Syspira sp. & 5.07 & 45 & 31 & 143 & 219 \\
\hline \multirow[t]{2}{*}{ Dictynidae } & Dictyna cholla Gertsch and Davis & 1.13 & 8 & 11 & 30 & 49 \\
\hline & Dictyna sp. & 1.94 & 15 & 14 & 55 & 84 \\
\hline \multirow[t]{3}{*}{ Filistatidae } & Filistatoides sp. & 0.18 & 4 & 1 & 3 & 8 \\
\hline & Kukulcania hibernalis Hentz & 0.02 & & 1 & & 1 \\
\hline & Kukulcania sp. & 0.3 & 5 & 7 & & 12 \\
\hline \multirow[t]{11}{*}{ Gnaphosidae } & Cesonia gertschi Platnich et Shadab & 0.12 & 1 & 1 & 3 & 5 \\
\hline & Drassyllus mirus Platnick et Shadab & 1.83 & 47 & 8 & 24 & 79 \\
\hline & Litopyllus realisticus (Chamberlin) & 0.02 & & 1 & & 1 \\
\hline & Micaria jeanae Gertsch & 0.05 & 1 & & 1 & 2 \\
\hline & Neosimiris pubecens (Banks) & 1.65 & 16 & 8 & 48 & 72 \\
\hline & Scopoides asceticum (Chamberlin) & 1.27 & 10 & 10 & 35 & 55 \\
\hline & Scopoides nesiotes (Chamberlin) & 0.53 & & 1 & 22 & 23 \\
\hline & Trachyzelotes jaxartensis (Kroneberg) & 0.1 & 2 & 2 & & 4 \\
\hline & Zelotes monachus Chamberlin & 0.02 & 1 & & & 1 \\
\hline & Zelotes catholicus Chamberlin & 0.05 & & 2 & & 2 \\
\hline & Zelotes ubicki Platnick et Shadab & 2.22 & 43 & 13 & 40 & 96 \\
\hline Homalonychidae & Homalonychus theologus Chamberlin & 0.5 & 1 & 5 & 14 & 20 \\
\hline Loxoscelidae & Loxosceles baja Gertsch et Ennik & 0.9 & 14 & 7 & 16 & 37 \\
\hline \multirow[t]{2}{*}{ Lycosidae } & Hogna carolinesis (Walckenaer) & 0.07 & 3 & & & 3 \\
\hline & Geolycosa sp. & 1.34 & & & 58 & 58 \\
\hline Oecobiidae & Oecobius isolatus Chamberlin & 0.4 & 1 & 5 & 10 & 16 \\
\hline \multirow[t]{4}{*}{ Oonopidae } & Scaphiela espera Chamberlin & 7.8 & 113 & 96 & 128 & 337 \\
\hline & Oonops stylifer Gertsch & 0.8 & 17 & 10 & 8 & 35 \\
\hline & Yumates nesophila Chamberlin & 1.02 & 21 & 13 & 10 & 44 \\
\hline & Oonops sp. & 13.7 & 161 & 166 & 271 & 598 \\
\hline Oxyopidae & Oxyopes tridens Brady & 2.33 & 15 & 8 & 78 & 101 \\
\hline Philodromidae & Apollophanes. sp. & 0.02 & & 1 & & 1 \\
\hline Pholcidae & Psilochorus pullulus (Hentz) & 0.2 & 2 & 1 & 5 & 8 \\
\hline Plectreuridae & Plectreurys bicolor Banks & 0.21 & 4 & 1 & 4 & 9 \\
\hline
\end{tabular}




\begin{tabular}{|c|c|c|c|c|c|c|}
\hline Familia & Especies & $\%$ & $\hat{0}$ & 움 & $J$ & Totales \\
\hline Salenopidae & Selenops sp. & 0.1 & & & 4 & 4 \\
\hline \multirow[t]{2}{*}{ Thomisidae } & Xysticus lassanus Chamberlin & 3.4 & 65 & 13 & 67 & 145 \\
\hline & Tmarus sp. & 0.02 & & & 1 & 1 \\
\hline \multirow[t]{7}{*}{ Salticidae } & Habronattus divaricatus (Banks) & 1.11 & 12 & 2 & 34 & 48 \\
\hline & Habronattus signatus (Banks) & 1.06 & 3 & 9 & 34 & 46 \\
\hline & Metacyrba sp. & 0.14 & 2 & 4 & & 6 \\
\hline & Metaphidippus sp. 1 & 0.23 & 2 & 7 & 1 & 10 \\
\hline & Metaphidippus sp. 2 & 0.06 & & 1 & 2 & 3 \\
\hline & Peckamia sp. & 0.05 & & 2 & & 2 \\
\hline & Sitticus sp. & 0.92 & 19 & 6 & 15 & 40 \\
\hline \multirow[t]{3}{*}{ Cyrtaucheniidae } & Actinoxia sp. & 0.02 & 1 & & & 1 \\
\hline & Myrmekiaphila sp. & 0.02 & 1 & & & 1 \\
\hline & Promyrmekiaphila sp. & 0.6 & 12 & & 12 & 24 \\
\hline \multirow[t]{2}{*}{ Dipluridae } & Euagrus josephus Chamberlin & 0.18 & 4 & 3 & 1 & 8 \\
\hline & Totales & 100 & 1102 & 771 & 2449 & 4322 \\
\hline
\end{tabular}

Cuadro 2. Diversidad estacional de la aracnofauna del Comitán B.C.S., México durante el período de 1991-1992

\begin{tabular}{lccccc}
\hline & \multicolumn{1}{c}{ Is } & & & $H^{\prime}$ \\
& Primavera & Verano & Otoño & Invierno & 3.49 \\
\hline Primavera & 1 & 0.98 & 0.95 & 0.94 & 3.334 \\
Verano & 0.92 & 1 & 0.98 & 0.83 & 3.451 \\
Otoño & 0.95 & 0.95 & 1 & 0.86 & 3.682 \\
Invierno & 0.94 & 0.94 & 0.86 & 1 & \\
\hline
\end{tabular}

$H^{\prime}=$ Índice de Shannon; Is = Coeficiente de similitud de Morisita.

\section{Discusión}

La gran abundancia de arañas en este estudio se debió a la presencia de la familia Miturgidae y específicamente a S. tigrina, que llega a representar el $50 \%$ de todas las arañas del suelo (Nieto y Jiménez, 2009). Probablemente $S$. tigrina sea una especie diplocrónica, es decir, que tiene 2 periodos reproductivos e inverna en estado adulto, ya que se encontraron tanto adultos como juveniles durante todo el año; además, su abundancia aumentó durante el veranootoño (Fig. 2 y 3), confirmando que tiene gran afinidad a áreas cálidas con humedad relativamente baja en suelos desnudos (Nieto y Jiménez, 2009). Esta especie tiene una gran adaptación a las zonas desérticas de Baja California, por su gran talla y su capacidad de desplazamiento (NietoCastañeda, 2004). Los individuos de S. tigrina fueron observados muy activos en suelos arenosos durante la noche y suelen confundirse con las arañas lobo (Lycosidae), por la similitud de sus movimientos; en el caso contrario estarían las Selenopidae por sus hábitos más sedentarios, lo que explica su baja presencia en las trampas, debido a que no son cursoriales o buscadoras activas. De acuerdo con el criterio de Costa (1991), es probable que individuos de Miturgidae compitan con las otras especies por refugios, así como por sus presas potenciales, logrando desplazar a individuos de menor tamaño, pero también es probable que no tenga ninguna competencia significativa, pues su máxima actividad se observó en otoño y la mayoría de las especies restantes tuvieron incrementos poblacionales principalmente en primavera y unas pocas en verano (Fig. 2). Al igual que en el caso de Miturgidae, una especie de Zodariidae (Diores triarmatus Lessert) fue numéricamente dominante en suelos del matorral xerófilo de la reserva de Kora en Kenya, debido a que estuvo asociada con la 
Cuadro 3. Variación mensual de las arañas del matorral sarcocaule de El Comitán, Baja California Sur, México, durante el ciclo anual de 1991-1992

\begin{tabular}{|c|c|c|c|c|c|c|c|c|c|c|c|c|}
\hline Especies & ENE & $F E B$ & $M A R$ & $A B R$ & $M A Y$ & $J U N$ & $J U L$ & $A G S$ & SEP & OCT & NOV & DIC \\
\hline Novalena sp & & & & & & & & & $\mathrm{x}$ & & & \\
\hline Tarsonops sectipes & $\mathrm{x}$ & $\mathrm{x}$ & $\mathrm{x}$ & $\mathrm{x}$ & $\mathrm{x}$ & & $\mathrm{x}$ & $\mathrm{x}$ & $\mathrm{x}$ & $\mathrm{x}$ & $\mathrm{x}$ & $\mathrm{x}$ \\
\hline Tarsonops sternalis & $\mathrm{x}$ & $\mathrm{x}$ & $\mathrm{x}$ & $\mathrm{x}$ & $\mathrm{x}$ & $\mathrm{x}$ & $\mathrm{x}$ & $\mathrm{x}$ & $\mathrm{x}$ & $\mathrm{x}$ & $\mathrm{x}$ & $\mathrm{x}$ \\
\hline Orthonops overtus & $\mathrm{x}$ & $\mathrm{x}$ & $\mathrm{x}$ & $\mathrm{x}$ & $\mathrm{x}$ & $\mathrm{x}$ & $\mathrm{x}$ & $\mathrm{x}$ & $\mathrm{x}$ & & $\mathrm{x}$ & \\
\hline Clubiona sp & & & $\mathrm{x}$ & $\mathrm{x}$ & $\mathrm{x}$ & $\mathrm{x}$ & $\mathrm{x}$ & $\mathrm{x}$ & $\mathrm{x}$ & $\mathrm{x}$ & $\mathrm{x}$ & $\mathrm{x}$ \\
\hline Syspira tigrina & $\mathrm{x}$ & $\mathrm{x}$ & $\mathrm{x}$ & $\mathrm{x}$ & $\mathrm{x}$ & $\mathrm{x}$ & $\mathrm{x}$ & $\mathrm{x}$ & $\mathrm{x}$ & $\mathrm{x}$ & $\mathrm{x}$ & $\mathrm{x}$ \\
\hline Syspira sp & $\mathrm{x}$ & $\mathrm{x}$ & $\mathrm{x}$ & $\mathrm{x}$ & $\mathrm{x}$ & $\mathrm{x}$ & $\mathrm{x}$ & $\mathrm{x}$ & $\mathrm{x}$ & $\mathrm{x}$ & $\mathrm{x}$ & $\mathrm{x}$ \\
\hline Castianeira dorsata & & & & $\mathrm{x}$ & & $\mathrm{x}$ & $\mathrm{x}$ & $\mathrm{x}$ & & & & \\
\hline Trachelas speciosus & & & & & $\mathrm{x}$ & & & & & & & \\
\hline Dictyna cholla & $\mathrm{x}$ & $\mathrm{x}$ & $\mathrm{x}$ & $\mathrm{x}$ & & & & $\mathrm{x}$ & $\mathrm{x}$ & & $\mathrm{x}$ & \\
\hline Dictyna sp & $\mathrm{x}$ & $\mathrm{x}$ & $\mathrm{x}$ & $\mathrm{x}$ & $\mathrm{x}$ & $\mathrm{x}$ & $\mathrm{x}$ & $\mathrm{x}$ & $\mathrm{x}$ & $\mathrm{x}$ & $\mathrm{x}$ & $\mathrm{x}$ \\
\hline Filistatoides sp. & & & $\mathrm{x}$ & $\mathrm{x}$ & $\mathrm{x}$ & & $\mathrm{x}$ & & & & $\mathrm{x}$ & $\mathrm{x}$ \\
\hline Kukulkania hibernalis & & & & & & $\mathrm{x}$ & & & & & & \\
\hline Kukulkania sp. & $\mathrm{x}$ & $\mathrm{x}$ & & $\mathrm{x}$ & & & $\mathrm{x}$ & & & & $\mathrm{x}$ & $\mathrm{x}$ \\
\hline Cesonia gertschi & & & & & $\mathrm{x}$ & & & & $\mathrm{x}$ & $\mathrm{x}$ & $\mathrm{x}$ & \\
\hline Drasyllus mirus & $\mathrm{x}$ & $\mathrm{x}$ & $\mathrm{x}$ & $\mathrm{x}$ & $\mathrm{x}$ & $\mathrm{x}$ & $\mathrm{x}$ & & $\mathrm{x}$ & & $\mathrm{x}$ & $\mathrm{x}$ \\
\hline Litophillus realisticus & & & & & & & $\mathrm{x}$ & & & & & \\
\hline Micaria jeanae & & & & & & & & & $\mathrm{x}$ & & $\mathrm{x}$ & \\
\hline Niosimiriz pubescens & $\mathrm{x}$ & $\mathrm{x}$ & $\mathrm{x}$ & $\mathrm{x}$ & $\mathrm{x}$ & $\mathrm{x}$ & $\mathrm{x}$ & $\mathrm{x}$ & $\mathrm{x}$ & $\mathrm{x}$ & $\mathrm{x}$ & $\mathrm{x}$ \\
\hline Scopoides asceticum & $\mathrm{x}$ & & $\mathrm{x}$ & $\mathrm{x}$ & $\mathrm{x}$ & $\mathrm{x}$ & $\mathrm{x}$ & $\mathrm{x}$ & $\mathrm{x}$ & $\mathrm{x}$ & $\mathrm{x}$ & $\mathrm{x}$ \\
\hline Scopoides nesiotes & & & $\mathrm{x}$ & & & & $\mathrm{x}$ & & & & & \\
\hline Trachyzeloges jaxartensis & & & & $\mathrm{x}$ & & & $\mathrm{x}$ & $\mathrm{x}$ & & & & \\
\hline Zelotes catholicus & $\mathrm{x}$ & & & & & & & & & & & \\
\hline Zelotes monachus & $\mathrm{x}$ & $\mathrm{x}$ & $\mathrm{x}$ & $\mathrm{x}$ & $\mathrm{x}$ & $\mathrm{x}$ & $\mathrm{x}$ & $\mathrm{x}$ & $\mathrm{x}$ & $\mathrm{x}$ & $\mathrm{x}$ & $\mathrm{x}$ \\
\hline Zeolotes ubicki & & & & & $\mathrm{x}$ & & & $\mathrm{x}$ & $\mathrm{x}$ & & & \\
\hline Homalonychus theologus & & $\mathrm{x}$ & $\mathrm{x}$ & $\mathrm{x}$ & $\mathrm{x}$ & $\mathrm{x}$ & $\mathrm{x}$ & $\mathrm{x}$ & $\mathrm{x}$ & $\mathrm{x}$ & $\mathrm{x}$ & \\
\hline Loxosceles baja & & & & $\mathrm{x}$ & $\mathrm{x}$ & $\mathrm{x}$ & $\mathrm{x}$ & $\mathrm{x}$ & $\mathrm{x}$ & $\mathrm{x}$ & $\mathrm{x}$ & $\mathrm{x}$ \\
\hline Hogna carolinensis & & & $\mathrm{x}$ & & & & $\mathrm{x}$ & $\mathrm{x}$ & & & & \\
\hline Geolycosa sp. & & & & & & & $\mathrm{x}$ & $\mathrm{x}$ & $\mathrm{x}$ & $\mathrm{x}$ & $\mathrm{x}$ & \\
\hline Oecobius isolatus & & $\mathrm{x}$ & & $\mathrm{x}$ & $\mathrm{x}$ & $\mathrm{x}$ & $\mathrm{x}$ & $\mathrm{x}$ & $\mathrm{x}$ & & $\mathrm{x}$ & $\mathrm{x}$ \\
\hline Scaphiella espera & $\mathrm{x}$ & $\mathrm{x}$ & $\mathrm{x}$ & $\mathrm{x}$ & $\mathrm{x}$ & $\mathrm{x}$ & $\mathrm{x}$ & $\mathrm{x}$ & $\mathrm{x}$ & $\mathrm{x}$ & $\mathrm{x}$ & $\mathrm{x}$ \\
\hline Yumates nesophila & $\mathrm{x}$ & $\mathrm{x}$ & $\mathrm{x}$ & $\mathrm{x}$ & $\mathrm{x}$ & $\mathrm{x}$ & $\mathrm{x}$ & $\mathrm{x}$ & $\mathrm{x}$ & $\mathrm{x}$ & $\mathrm{x}$ & $\mathrm{x}$ \\
\hline Oonops styliffer & $\mathrm{x}$ & $\mathrm{x}$ & $\mathrm{x}$ & $\mathrm{x}$ & $\mathrm{x}$ & & $\mathrm{x}$ & $\mathrm{x}$ & $\mathrm{x}$ & & $\mathrm{x}$ & $\mathrm{x}$ \\
\hline Oonops sp. & $\mathrm{x}$ & $\mathrm{x}$ & $\mathrm{x}$ & $\mathrm{x}$ & $\mathrm{x}$ & $\mathrm{x}$ & $\mathrm{x}$ & $\mathrm{x}$ & $\mathrm{x}$ & $\mathrm{x}$ & $\mathrm{x}$ & $\mathrm{x}$ \\
\hline Oxyopes tridens & $\mathrm{x}$ & $\mathrm{x}$ & $\mathrm{x}$ & $\mathrm{x}$ & $\mathrm{x}$ & $\mathrm{x}$ & $\mathrm{x}$ & $\mathrm{x}$ & $\mathrm{x}$ & $\mathrm{x}$ & $\mathrm{x}$ & $\mathrm{x}$ \\
\hline Apollophanes sp. & $\mathrm{x}$ & $\mathrm{x}$ & & & $\mathrm{x}$ & & & & & & $\mathrm{x}$ & $\mathrm{x}$ \\
\hline Psilochorus pulullus & & $\mathrm{x}$ & & $\mathrm{x}$ & & $\mathrm{x}$ & $\mathrm{x}$ & & & & & $\mathrm{x}$ \\
\hline
\end{tabular}




\begin{tabular}{|c|c|c|c|c|c|c|c|c|c|c|c|c|}
\hline Especies & $E N E$ & $F E B$ & $M A R$ & $A B R$ & $M A Y$ & $J U N$ & $J U L$ & $A G S$ & SEP & $O C T$ & $\mathrm{NOV}$ & $D I C$ \\
\hline Plectreuris bicolor & $\mathrm{x}$ & & $\mathrm{x}$ & $\mathrm{x}$ & & & & & $\mathrm{x}$ & & & $\mathrm{x}$ \\
\hline Selenops sp. & & $\mathrm{x}$ & $\mathrm{x}$ & & $\mathrm{x}$ & & & & & & & \\
\hline Xysticus lassanus & $\mathrm{x}$ & $\mathrm{x}$ & $\mathrm{x}$ & $\mathrm{x}$ & $\mathrm{x}$ & $\mathrm{x}$ & $\mathrm{x}$ & & $\mathrm{x}$ & $\mathrm{x}$ & $\mathrm{x}$ & $\mathrm{x}$ \\
\hline Tmarus sp. & & & & $\mathrm{x}$ & & & & & & & & \\
\hline Habronattus divaricatus & & $\mathrm{x}$ & $\mathrm{x}$ & $\mathrm{x}$ & $\mathrm{x}$ & $\mathrm{x}$ & $\mathrm{x}$ & $\mathrm{x}$ & $\mathrm{x}$ & $\mathrm{x}$ & & $\mathrm{x}$ \\
\hline Habronattus signatus & & $\mathrm{x}$ & $\mathrm{x}$ & $\mathrm{x}$ & $\mathrm{x}$ & $\mathrm{x}$ & $\mathrm{x}$ & $\mathrm{x}$ & $\mathrm{x}$ & $\mathrm{x}$ & $\mathrm{x}$ & $\mathrm{x}$ \\
\hline Metacyrba sp. & & & & $\mathrm{x}$ & & & $\mathrm{x}$ & $\mathrm{x}$ & $\mathrm{x}$ & $\mathrm{x}$ & $\mathrm{x}$ & \\
\hline Metaphidippus sp.1 & & & & $\mathrm{x}$ & & & $\mathrm{x}$ & $\mathrm{x}$ & & & & \\
\hline Metaphidippus sp. 2 & & & & & & & & & $\mathrm{x}$ & & & \\
\hline Peckamia sp. & & & & & & & & $\mathrm{x}$ & & $\mathrm{x}$ & & \\
\hline Sitticus sp. & & & & $\mathrm{x}$ & $\mathrm{x}$ & $\mathrm{x}$ & $\mathrm{x}$ & $\mathrm{x}$ & $\mathrm{x}$ & $\mathrm{x}$ & & \\
\hline Actonoxia sp. & & & & & & & $\mathrm{x}$ & & & & & \\
\hline Mymekiaphila sp. & & & & & & & $\mathrm{x}$ & & & & & \\
\hline Promyrmekiaphila $s p$ & $\mathrm{x}$ & & & & & & & & & & & $\mathrm{x}$ \\
\hline Euagrus josephus & & $\mathrm{x}$ & $\mathrm{x}$ & & & & & & & $\mathrm{x}$ & $\mathrm{x}$ & \\
\hline
\end{tabular}

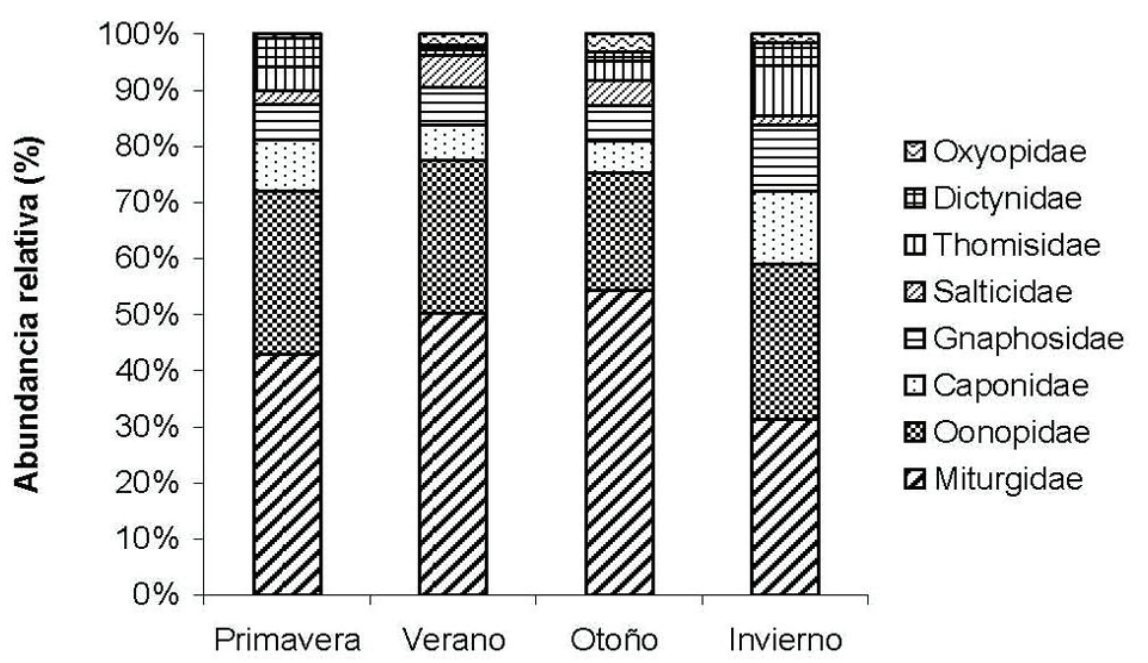

Figura 3. Fluctuación estacional de las familias más abundantes de arañas del suelo en El Comitán, B.C.S., México.

abundancia de hormigas en los hábitats secos y de las cuales se alimentó (Russell-Smith et al., 1987). Aunque se desconocen los hábitos alimentarios de $S$. tigrina, esta situación pudiera ser similar en el matorral xerófilo de la localidad en estudio, donde estaría aprovechando el recurso más abundante.

Platnick (1995) establece que los géneros Orthonops y Scaphiella tienden a compartir microhábitats del suelo, teniendo una gran semejanza en su coloración.
Las Oonopidae también se han registrado como especies muy abundantes en matorrales semideciduos en Nigeria, siendo la familia más diversa, seguida de Gnaphosidae y Salticidae (RussellSmith et al., 1987), como en este estudio. En El Comitán el suelo es llano, de consistencia arenosa y las rocas son muy escasas, por lo que las arañas del suelo como las Caponiidae y Homalonychidae aprovechan a manera de microhábitat para resguardarse los troncos de cactos en descomposición, como los del cardón (Pachycereus pringlei) (Domínguez y Jiménez 2005). Asimismo, en época de secas (primavera-verano) hay un aumento de hojarasca y pasto seco que también pudiera proveer una mayor cantidad de microhábitats para las arañas de los géneros Scaphiella y Oonops. Es pertinente mencionar que a las Caponiidae y las Oonopidae se les ha encontrado en nidos de ratas (Neotoma spp.) en varias localidades de California (Ryckman y Lee, 1956; Vetter y Prentice, 2002), pues esos nidos están constituidos de materiales muy diversos y abundantes que les proporcionan a estas arañas un excelente refugio para su sobrevivencia, por lo 
que no se descarta que en la localidad de estudio, ambas familias lleguen a ocupar esos sitios.

Las familias Gnaphosidae y Salticidae fueron las que agruparon el mayor número de especies, lo que era de esperarse, pues se conoce que están bien representadas en los desiertos del mundo (Polis y Yamashita, 1991). En comunidades áridas de Nuevo Mexico, las Gnaphosidae han sido registradas como las más diversas (Abraham, 1983; Muma, 1975, 1980a), pero al igual que las Zodariidae, las Gnaphosidae y Salticidae por su abundancia, también llegan a ser numéricamente dominantes en algunos desiertos de América y sabanas y desiertos de África (Fautin, 1946; Muma, 1975; Russell-Smith 2002; RusselSmith et al., 1987). El dominio de las Gnaphosidae en zonas áridas probablemente se debe a que están mejor adaptadas a las zonas secas que las Salticidae (Russell-Smith, 2002). Con excepción de Trachyzelotes jaxartensis (Kroneberg), especie del mediterráneo que ha sido introducida a Baja California (Platnick y Murphy, 1984), todas las especies de esta familia son endémicas de la península; sin embargo, es pertinente mencionar que Zelotes ubicki Platnick y Shadab, fue la especie más abundante, mientras que $Z$. monachus y L. realisticus fueron las más escasas. En las Salticidae, destaca el género Habronattus por agrupar especies propias de los suelos desérticos (Griswold, 1987), lo que coincide con los resultados de este estudio, donde las 2 especies encontradas fueron de las más abundantes (Cuadro 1).

Las arañas errantes de las familias Homalonychidae, Philodromidae, Plectreuridae, Selenopidae y Cyrtauchenidae fueron escasamente capturadas, a causa de sus hábitos más sedentarios. Algunas de ellas tienen desplazamientos limitados, como Homalonychus theologus Chamberlin que se ha recolectado bajo cardones en descomposición (Domínguez y Jiménez, 2005) o Selenops sp. y Plectreurys bicolor Banks que se localizaron bajo cortezas o rocas en el suelo. Las Cyrtauchenidae permanecieron en sus madrigueras, sólo se les capturó después de una fuerte lluvia (invierno), cuando el suelo se anegó y sus refugios se inundaron, y durante la época reproductiva, cuando los machos se expusieron para buscar hembras para el apareamiento. Esto explica su baja presencia en las trampas, pues no son arañas cursoriales, lo que sugiere que tienen un comportamiento sedentario y explica por qué estas arañas no son capturadas con tanta frecuencia (Costa et al., 1991).

La escasez en la captura de individuos de Agelenidae, Filistatidae, Dipluridae, Loxoxcelidae, Pholcidae y Oecobiidae se debió a sus hábitos de caza, pues se trata de especies tejedoras que sólo se retiran un poco de sus redes para perseguir a sus presas (Riechert, 1974). Estas arañas están más o menos limitadas a las dimensiones de sus redes y pueden ocupar microhábitats bajo cortezas o cactos en descomposición, pero no se descarta la posibilidad de que invadan las madrigueras de roedores u otras cavidades, como lo hacen Artema atlanta Walckenaer (Pholcidae) y Latrodectus hesperus Chaberlin e Ivie (Theridiidae) en el desierto de Chihuahua (Fowler y Whitford, 1985) y Psilochorus imitatus Gertsch y Mulaik (Pholcidae) en pastos de zonas áridas de Nuevo México (Muma, 1980b), donde llegan a ser dominantes.

Duffey (1972) sostiene que las arañas adultas no superan el $48 \%$ de su abundancia; mientras que en trampas enterradas alcanzan proporciones cercanas al 80\% (Costa et al., 1991). La abundancia relativa de Thomisidae y Oxyopidae durante el invierno y de Dictynidae durante la primavera se debió a la presencia de Xysticus lassanus Chamberlin, Dictyna spp. (Dictynidae) y O. tridents (Oxyopidae). Oxyopes tridents, una especie del suelo y de hábitos diurnos (Brady, 1964), la registran Coyle (1981) y Corey y Taylor (1988) como especie esporádica, resultados que difieren de los que aquí se presentan, ya que en este trabajo fue relativamente abundante.

En el presente estudio, la proporción de adultos y juveniles se mantuvo más o menos estable, al representar los adultos un total de 43\%; sin embargo, los repentinos incrementos de algunas familias (Miturgidae, Salticidae, Thomisidae, Dictynidae y algunas Gnaphosidae y Caponiidae) se debieron a que los adultos y juveniles de sus especies aparecieron en gran número después de un periodo durante el cual fueron escasos o ausentes. No se sabe si estos incrementos se deban a que los juveniles o los huevos hibernen, o bien, que se trate de organismos diplocronos, es decir, que tengan 2 periodos reproductivos y que entren en diapausa en el estado adulto.

El dominio de los machos con respecto a las hembras estuvo relacionado con la actividad de búsqueda de parejas (1 107 machos / 764 hembras). En las Mygalomorphae fue más evidente, ya que sólo se presentaron 3 hembras por 18 machos. La búsqueda de pareja para el apareamiento influye en la susceptibilidad de captura, como en Miturgidae, Gnaphosidae y Thomisidae entre otras.

Es importante considerar la capacidad de desplazamiento de las arañas errantes,que tienen mejores oportunidades para seleccionar entre un mayor número de hábitats y mayor variedad de presas, pues una dieta más amplia debe ser más estable que una dieta restringida (Krebs 1895). Esta capacidad también les permite realizar una selección de microhábitat mayor, aprovechando la gran cantidad de microclimas, como el proporcionado por la hojarasca, ya que no sólo las protege de las fluctuaciones de temperatura externa, sino también de la desecación (Edgar y Leonen en: Foelix, 1982). Estos factores y la provisión de presas reducen la depredación interespecífica y permiten la persistencia de especies raras (Uetz, 1976). Los diferentes ciclos de vida de las arañas dan lugar a la diversidad 
de especies en un hábitat determinado y si hay mayor diversidad, entonces también se promueve la ocupación de diferentes microhábitats por diferentes poblaciones dentro de ese mismo hábitat (Dondale, 1977).

Otro factor que probablemente influyó en la diversidad fue la hojarasca, ya que la diversidad de arañas aumenta con la profundidad de la misma (Bultman et al., 1982). La hojarasca proporciona un gran número de microhábitats para las arañas (Uetz, 1976), y esto es de gran importancia si tomamos en cuenta las múltiples variaciones espaciales y temporales de microclimas que pueden existir en este hábitat.

La diversidad alcanzada en las 4 estaciones del año fue alta y se mantuvo más o menos estable a lo largo del tiempo, observándose un ligero incremento en el invierno que coincide con la disminución de los individuos de la especie dominante $S$. tigrina (Cuadro 1 y 3). En la zona de estudio se presenta un clima cálido casi estable durante la mayor parte del año, lo que seguramente favorece la estabilidad de las poblaciones de arañas cazadoras en la zona. Sin embargo, las lluvias durante el invierno pueden alterar estas poblaciones de manera considerable, como se observó en la disminución del número de arañas durante esta estación (Fig. 2).

En el desierto de El Comitán se encontró un número de especies que se consideran raras porque presentaron bajas abundancias o porque fueron poco frecuentes en ciertas épocas o meses del año (T. speciosus, L. realisticus, $Z$. catholicus, Tmarus sp, Metaphidippus sp. 2), pero siempre con suficiente traslapamiento con otras especies más abundantes y que en conjunto mantuvieron la diversidad estable durante todo el año (Dean et al., 1982). Esto se puede atribuir a factores ecológicos de competencia interespecífica, a que no estén en su hábitat óptimo, o bien, a efectos poblacionales, presentando densidades bajas en su área de distribución (Rabinowitz y Coli en Ezcurra, 1990).

Magurran (1988) y Krebs (1985) afirman que en una comunidad desértica, la mayoría de las especies son raras, y sólo algunas son muy abundantes, como es éste el caso, pero Krebs (1985) considera que los desiertos son comunidades relativamente sencillas, a pesar de que permiten una diversidad sorprendente de especies animales, como muestran los resultados del estudio en esta comunidad de arañas.

\section{Agradecimientos}

A los revisores anónimos por sus atinados comentarios a este manuscrito. Este trabajo fue apoyado por el Centro de Investigaciones Biológicas del Noroeste, S.C.

\section{Literatura citada}

Abraham, B. 1983. Spatial and temporal patterns in a sagebrush steppe spider community. Journal of Arachnology 11:31-50.

Allred, D., D. Beck y C. Jorgensen. 1963. Biotic communities of the Nevada Test Site. Brigham Young University Science Bulletin 2:1-52.

Axelrod, D. I. 1979. Age and origin of Sonoran Desert vegetation. Occasional Papers of the California Academy of Sciences 132:1-74.

Brady, A. R. 1964. The lynx spider of North American, North of Mexico (Araneae:Oxyopidae). Bulletin of the Museum of Comparative Zoology 131:429-518.

Bultman, T. L., G. W. Uetz y A. R. Brady. 1982. A comparison of cursorial spider communities along a succesional gradient. Journal of Arachnology 10:23-33.

Chew, R. M. 1961. Ecology of spiders of a desert community. Journal of the New York Entomological Society 69:5-41.

Cloudsley-Thompson, J. L. 1979. El hombre y la biología de zonas áridas. Blume Ecología, Barcelona. 255 p.

Cloudsley-Thompson, J. L. 1983. Desert adaptation in spiders. Journal of Arid Environments 6:307-317.

Coyle, F. A. 1981. Effects of clear cutting on the spider community of a southern Apalachian forest. Journal of Arachnology 9:285-298.

Corey, D. T. y W. K. Taylor. 1988. Ground surface spiders in three central Florida plant communities. Journal of Arachnology 16:213-221.

Costa, F. G. 1991. Fenología de Lycosa malitosa Tullgreen (Araneae, Lycosidae) como componente del critozoos en Marindia, localidad costera del sur del Uruguay. Boletín de la Sociedad Zoológica del Uruguay 2a época 6:8-21.

Costa, F. G., F. Pérez-Miles, E. Gudyan, L. Prandi y R. M. Capocasale. 1991. Ecología de los arácnidos criptozoicos, excepto ácaros, de la sierra de las Minas (Uruguay). Órdenes y Familias. Aracnología 13:1-41.

Dean, D. A., W. L. Sterling y N. V. Horner. 1982. Spiders in eastern Texas cotton field. Journal of Arachnology 10:251260.

Domínguez, K. y M. L. Jiménez. 2005. Mating and self-burying behavior of Homalonychus theologus Chamberlin (Araneae, Homalonychidae) in Baja California Sur. The Journal of Arachnology 33:167-174.

Domínguez, K. y M. L. Jiménez. 2008. Composition of spider prey captured by wasp Trypoxylon (Trypargilum) tridentatum tridentatum in two habitats in an oasis in Baja California Sur, México. Canadian Entomologist 140:388-392.

Dondale, C. D., 1977. Life histories and distribution patterns of hunting spiders (Araneae) in an Ontario meadow. The Journal of Arachnology. 4:73-93.

Duffey, E. 1972. Ecological survey and the arachnologists. Bulletin of the British Arachnological Society 2:69-82.

Ezcurra, E. 1990. ¿Por qué hay tantas especies raras? La riqueza y rareza biológicas en las comunidades naturales. Ciencias - especial - 4:82-88.

Fautin, R. W. 1946. Biotic communities of the northern desert shrub biome in western Utah. Ecological Monographs 16:251-310. 
Foelix, R. 1996. Biology of spiders, segunda edición. Oxford University Press, New York. 330 p.

Fowler, H. y W. Whitford. 1985. Structure and organization of a winter community of cavity-inhabiting, web-building spiders (Pholcidae and Theridiidae) in a Chihuahua desert habitat. Journal of Arid Environments 8:57-65.

García, E. 1981. Modificaciones al sistema de clasificación climática de Köppen. Instituto de Geografía, UNAM, México, D.F. 246 p.

Griswold, C. E. 1987. A revision of the jumping spider genus Habronattus F.O.P.-Cambridge (Araneae:Salticidae) with phenetic and cladistic analyses, University of California Publications in Entomology, vol. 107. University of California Press, Berkeley. 344.p.

Hammond, E. H. 1954. A geomorphic study of the Cape Region of Baja California. University of California Press, Berkeley, $94 \mathrm{p}$.

Jiménez, M. L. 1996. El orden Araneae. In Biodiversidad, taxonomía y biogeografía de los artrópodos de México: hacia una síntesis de su conocimiento, J. Llorente Bousquets, A. N. García-Aldrete y E. González-Soriano (eds.). Comisión Nacional para el Conocimiento y Uso de la Biodiversidad / Universidad Nacional Autónoma de México, México, D.F. p. 83-101.

Jiménez, M. L. 1998. Aracnofauna asociada a las viviendas de la ciudad de La Paz, B.C.S., México. Folia Entomológica Mexicana 102:1-10.

Jiménez, M. L. y A. Tejas. 1994. Las arañas presa de la avispa lodera Trypoxylon (Trypargilum) tridentatum tridentatum en Baja California Sur, México. Southwest Entomologist 19:173-179.

Jiménez, M. L. y A. Tejas. 1996. Variación temporal de la araneofauna en frutales de la región del Cabo, Baja California Sur. México. Southwestern Entomolology 21:331-335.

Jiménez, M. L., R. Servín, A. Tejas y R. Aguilar. 1992. La composición de presas de la avispa lodera. Sceliphron jamaicense lucae en la Región del Cabo, México. Southwest Entomologist 17:169-180.

Krebs, C. J. 1985. Ecología, estudio de la distribución y abundancia. Harla, México. 753p.

Llinas-Gutíerrez, J. y M. L. Jiménez. 2004. Arañas de humedales del sur de Baja California, México. Anales del Instituto de Biología, Universidad Nacional Autónoma de México, Serie Zoología 75:283-302.

León de la Luz, J. L, R. Coria y M. Cruz. 1996. Fenología floral de una comunidad árido-tropical de Baja California Sur, México. Acta Botanica Mexicana 35:45-64.

Nieto-Castañeda, I. G. 2004. Arañas edáficas (Caponiidae, Gnaphosidae,Lycosidae,MiturgidaeyOonopidae(Arachnida: Araneae) asociadas a humedales de Baja California Sur, México. Tesis maestría, Centro de Investigaciones Biológicas del Noroeste, La Paz, B.C.S. 111 p.

Nieto-Castañeda, I. G. y M. L Jiménez-Jiménez. 2009. Posible niche differentiation of two desert wandering spiders of the genus Syspira (Araneae:Miturgidae). Journal of Arachnology
37:299-305.

Magurran, A .E. 1988. Ecological diversity and its measurement. Cambridge University Press, Cambridge. 179 p.

Muma, M. H. 1975. Long-term can trapping for population analyses of ground-surface, arid-land arachnids. Florida Entomologist 58:257-270.

Muma, M. H., 1980a. Comparison of three methods for estimating solpugid (Arachnida) populations. Journal of Arachnology 8:267-270.

Muma, M. H. 1980b. Comparison of ground-surface spider populations in pinyon-juniper and arid-grass land association in southwestern New México. Florida Entomologist 63:211222.

Platnick, N. I. 1995. A revision of the spider genus Orthonops (Araneae, Caponiidae). American Museum Novitates 3150:1-18.

Platnick, N. I. y J. A. Murphy. 1984. A revision of the spider genera Trachyzelotes and Urozelotes (Araneae, Gnaphosidae). American Museum Novitates 2792:1-30.

Polis, G.A. y S.J. McCormick. 1986. Scorpions, spiders and solpugids: predation and competition among distantly related taxa. Oecologia 71:111-116.

Polis, G. A. y T. Yamashita. 1991. The ecology and importante of predaceous arthropods in desert communities, capítulo 7. In The ecology of desert communities, G. A. Polis (ed.). The University of Arizona Press, Tucson. p. 180-222.

Riechert, S. E. 1974. The pattern of local web distribution in a desert spider: mechanisms and seasonal variation. Journal of Animal Ecology 43:433-746.

Russell-Smith, A. 2002. A comparison of the diversity and composition of the ground-active spiders in Mkomazi Game Reserve, Tanzania and Etosha National Park, Namibia. Journal of Arachnology 30:383-388.

Russell-Smith, A., J. M. Ritchie y N. M. Collins. 1987. The surface-active spider fauna of arid bushland in Kora Reserve, Kenya. Bulletin of British Arachnological Sociedty 7:171174.

Ryckman, R. E. y D. Lee. 1956. Spider and phalangids associated with mammals (Citellus and Neotoma) in southwestern United States and Northen México. Annals of the Entomololgical Society of America 49:406-409.

SPP (Secretaría de Programación y Presupuesto) 1981a. Carta edafológica 1:1,000,000. Hoja La Paz, Dirección General de Geografía del Territorio Nacional, México, D.F.

SPP (Secretaría de Programación y Presupuesto). 1981b. Carta de climas 1:1,000,000. Hoja La Paz, Dirección General de Geografía del Territorio Nacional, México, D.F.

Uetz, G.W. 1976. Gradient analysis of spider communities in stream-side forest. Oecologia. 22:373-385.

Uetz, G. W. y J. D. Unzicker. 1976. Pitfall trapping in ecological studies of wandering spiders. Journal of Arachnology. 3:101111.

Vetter, R. S. y T. R. Prentice. 2002. The spider fauna associated with litter under woodrat middens in southern California (Arachnida: Araneae). Pan-Pacific Entomologist 78:23-33. 